\title{
Normally Regular Digraphs
}

\author{
Leif K. Jørgensen \\ Dept. of Mathematical Sciences \\ Aalborg University \\ Fr. Bajers Vej 7, 9220 Aalborg, Denmark. \\ leif@math.aau.dk \\ Submitted: Nov 1, 2014; Accepted: Oct 16, 2015; Published: Oct 30, 2015 \\ Mathematics Subject Classifications: 05E30, 05B05, 05C20, 05C50
}

\begin{abstract}
A normally regular digraph with parameters $(v, k, \lambda, \mu)$ is a directed graph on $v$ vertices whose adjacency matrix $A$ satisfies the equation $A A^{\mathrm{t}}=k I+\lambda\left(A+A^{\mathrm{t}}\right)+$ $\mu\left(J-I-A-A^{\mathrm{t}}\right)$. This means that every vertex has out-degree $k$, a pair of nonadjacent vertices have $\mu$ common out-neighbours, a pair of vertices connected by an edge in one direction have $\lambda$ common out-neighbours and a pair of vertices connected by edges in both directions have $2 \lambda-\mu$ common out-neighbours. We often assume that two vertices can not be connected in both directions.

We prove that the adjacency matrix of a normally regular digraph is normal. A connected $k$-regular digraph with normal adjacency matrix is a normally regular digraph if and only if all eigenvalues other than $k$ are on one circle in the complex plane. We prove several non-existence results, structural characterizations, and constructions of normally regular digraphs. In many cases these graphs are Cayley graphs of abelian groups and the construction is then based on a generalization of difference sets.

We also show connections to other combinatorial objects: strongly regular graphs, symmetric 2-designs and association schemes.
\end{abstract}

\section{Introduction}

In this section we introduce normally regular digraphs and other basic concepts. In Section 2 we show that complements of normally regular digraphs are normally regular and we prove bounds on the parameters. In Section 3 we prove that the adjacency matrices of normally regular digraphs are normal and we give a Bruck-Ryser type condition for existence. In Section 4 we characterize normally regular digraphs with $\mu=0$ or $\mu=k$. We consider eigenvalues of normally regular digraphs in Section 5 and show that a regular digraph with normal adjacency matrix is a normally regular digraph if and only if the non-trivial eigenvalues are on a circle in the complex plane. In Section 6 we consider 
relations to association schemes. The subject of Section 7 is partitions of the vertex set and in particular group divisible digraphs, i.e., orientations of complete multipartite graphs. In Section 8 we exclude existence for some parameter sets with small $\lambda$. Section 9 describes applications of normally regular digraphs to partitions of designs in smaller designs. In Section 10 we give several constructions of normally regular digraphs, primarily constructions as Cayley graphs. In particular, if $4 t+1,4 s+3$ and $q$ are prime powers and $r$ is not divisible by 3 we get normally regular Cayley digraphs with the following parameters

$$
\begin{gathered}
((4 t+1)(4 s+3),(4 t+2)(2 s+1), 4 s t+3 s+t+1) \\
((4 s+3)(2 s+1), 4 s+1, s, 1) \\
\left(\frac{q^{2 r}+q^{r}+1}{q^{2}+q+1}, q^{r}-q, q^{2}, q^{2}+q+1\right)
\end{gathered}
$$

and, if $q \equiv 1 \bmod 3$

$$
\left(\frac{1}{3}\left(q^{2}+q+1\right), q-1,1,3\right)
$$

The adjacency matrix of a digraph with vertex set $\left\{x_{1}, \ldots x_{v}\right\}$ is a $v \times v$ matrix $A$ in which the $(i, j)$-entry is

$$
A_{i j}= \begin{cases}1 & \text { if there is an edge directed from } x_{i} \text { to } x_{j} \\ 0 & \text { otherwise }\end{cases}
$$

Thus any square $\{0,1\}$-matrix is the adjacency matrix of a digraph if and only if all its diagonal entries are 0 . In this paper we consider such matrices that satisfy an equation involving $A A^{\mathrm{t}}$, where $A^{\mathrm{t}}$ denotes the transpose of $A$. The $(i, j)$ entry of $A A^{\mathrm{t}}$ (respectively $A^{\mathrm{t}} A$ ) is the number of common out-neighbours (respectively in-neighbours) of $x_{i}$ and $x_{j}$.

We say that a digraph is normal if its adjacency matrix $A$ is normal, i.e., if $A A^{\mathrm{t}}=A^{\mathrm{t}} A$. It follows that a digraph is normal if and only if for any two (not necessarily distinct) vertices $x$ and $y$ the number of common out-neighbours of $x$ and $y$ is equal to the number of common in-neighbours of $x$ and $y$.

We will use the notation $x \rightarrow y$ if there is an edge directed from $x$ to $y$ (and possibly also an edge from $y$ to $x$ ). If $x \rightarrow y$ then we say that $x$ dominates $y$. We write $x \leftrightarrow y$ if $x \rightarrow y$ and $x \leftarrow y$, and identify these two directed edges with an undirected edge.

The set $\{y \mid x \rightarrow y\}$ of out-neighbours of a vertex $x$ is denoted by $x^{+}$. Similarly $x^{-}$ denotes the set of in-neighbours. $d^{+}(x)=\left|x^{+}\right|$and $d^{-}(x)=\left|x^{-}\right|$denotes the out-degree and in-degree of $x$, respectively.

We will now introduce normally regular digraphs. We first give a matrix free definition. 
Definition 1. A normally regular digraph with parameters $(v, k, \lambda, \mu)$, also denoted by $\operatorname{NRD}(v, k, \lambda, \mu)$, is a directed graph on $v$ vertices such that

- every vertex has out-degree $k$

- any pair of non-adjacent vertices have exactly $\mu$ common out-neighbours,

- any pair of vertices $x, y$ such that exactly one of the edges $x \rightarrow y$ or $x \leftarrow y$ is present have exactly $\lambda$ common out-neighbours,

- any pair of vertices $x, y$ such that $x \leftrightarrow y$ have exactly $2 \lambda-\mu$ common out-neighbours.

A normally regular digraph (or any digraph) is said to be asymmetric if there is no pair $x, y$ such that $x \leftrightarrow y$.

Another very important property of a normally regular digraph, which will be proved later (Corollary 11) is that an $\operatorname{NRD}(v, k, \lambda, \mu)$ also satisfies that every vertex has in-degree $k$ and that the number of common in-neighbours of two vertices is equal to the number of common out-neighbours.

This definition may be stated in terms of the adjacency matrix.

Proposition 1. A $v \times v\{0,1\}$-matrix $A$ is the adjacency matrix of a normally regular digraph if and only if every diagonal entry is 0 and

$$
A A^{t}=k I+\lambda\left(A+A^{t}\right)+\mu\left(J-I-A-A^{t}\right),
$$

where $I$ is the identity matrix and $J$ is the matrix in which all entries are 1 . This normally regular digraph is asymmetric if and only if $A+A^{t}$ is a $\{0,1\}$ matrix.

It is important to note that a regular digraph with a normal adjacency matrix is not necessarily a normally regular digraph.

The author first intended to study only asymmetric normally regular digraphs. However, most of the results hold in the general case, so we will usually not assume that graphs are asymmetric, but for connections to association schemes and similar results we need to assume that the graph is asymmetric.

Asymmetric normally regular digraphs with $\mu=\lambda$ have been studied by Ito [12], [13], [14], [15] and [16], and also by Ionin and Kharaghani [11].

Fossorier, Ježek, Nation and Pogel [6] introduced what they call ordinary graphs. Their definition is similar to our Definition 1, but the number of common out-neighbours (and common in-neighbours) of $x$ and $y$ in the three cases is $a, b$ and $c$, respectively. They do not assume that $c=2 b-a$ (although this is satisfied in some of their results). Note that the equation $c=2 b-a$ is essential for the formulation of the definition of a normally regular digraph as a matrix equation, and thus it is essential for the theory.

U. Ott [28] considered Cayley graph construction from "generalized difference set" that leads to normally regular digraphs.

Another variation of normally regular digraphs is Deza digraphs. A regular digraph is said to be a Deza digraph if the number of common out-neighbours of two vertices is 
either $b$ or $c$, for some constants $b$ and $c$, but it need not depend on whether the vertices are adjacent or not. Deza digraphs have been studied by Wang and Feng [34].

Many constructions of normally regular digraphs use Cayley graphs of a group. Let $G$ be a group and let $S$ be a subset of $G$ not containing the group identity, which we denote by 1 . Then the Cayley graph $\operatorname{Cay}(G, S)$ is the graph whose vertices are the elements of $G$ and with edge set

$$
\left\{x \rightarrow y \mid x^{-1} y \in S\right\} .
$$

Let $S^{(-1)}=\left\{s^{-1} \mid s \in S\right\}$. Then Cay $(G, S)$ is undirected if $S^{(-1)}=S$ and asymmetric if $S^{(-1)} \cap S=\varnothing$.

Proposition 2. The Cayley graph $\operatorname{Cay}(G, S)$ is a normally regular digraph if and only if for every $g \in G, g \neq 1$, the number of pairs $(x, y) \in S \times S$ satisfying $y x^{-1}=g$ is $\mu$ if $g \notin S \cup S^{(-1)}, \lambda$ if $g$ is in exactly one of the sets $S, S^{(-1)}$ and $2 \lambda-\mu$ if $g \in S \cap S^{(-1)}$.

In [21] we will study normally regular digraphs constructed as Cayley graphs of abelian groups, and in [22] we present a table of small feasible parameters, enumerate small normally regular digraphs and prove some results related these graphs. In [23], group divisible normally regular digraphs, i.e, the digraphs considered in Section 4 and Section 7, are investigated.

It is well-known that, by definition, a strongly regular graph with parameters $(v, k, \lambda, \mu)$ is an undirected graph with $v$ vertices in which

- every vertex has degree $k$

- any pair of adjacent vertices have exactly $\lambda$ common neighbours

- any pair of non-adjacent vertices have exactly $\mu$ common neighbours.

Equivalently, a strongly regular graph is a graph whose adjacency matrix $A$ satisfies

$$
A^{2}=k I+\lambda A+\mu(J-I-A) \quad \text { and } \quad A J=J A=k J .
$$

Thus any normally regular digraph where all edges are undirected (i.e., $x \rightarrow y$ if and only if $x \leftarrow y$ ) is a strongly regular graph. Note however that we use $\lambda$ in a different meaning. For a normally regular digraph we will use $\lambda_{2}=2 \lambda-\mu$ to denote the number of common out-neighbours of a pair of vertices joined by two edges.

In the theory of normally regular digraphs we will require that $\lambda$ is an integer and thus $\mu$ and $\lambda_{2}$ are congruent modulo 2 . Thus not every strongly regular graph is a normally regular digraph.

There are some directed analogues of strongly regular graphs other than normally regular digraphs. Duval [5] introduced directed strongly regular graphs which have adjacency matrix $A$ satisfying

$$
A^{2}=t I+\lambda A+\mu(J-I-A) \text { and } A J=J A=k J .
$$


Many proof techniques from strongly regular graphs, especially the use of eigenvalues, are more easily applied to directed strongly regular graphs than to normally regular graphs, see [5] or [18].

Another well-known combinatorial structure to which normally regular digraphs are related are 2-designs (or Balanced Incomplete Block Designs). A $2-(v, k, \lambda)$ design is an incidence structure with $\{0,1\}$ incidence matrix $N$ of size $v \times b, b=\frac{\lambda v(v-1)}{k(k-1)}$ satisfying

$$
N N^{\mathrm{t}}=(k-\lambda) I+\lambda J .
$$

A 2-design is said to be symmetric if $b=v$. The parameter $k-\lambda$ is called the order of the symmetric design. For information on design theory, see Beth, Jungnickel and Lenz [2].

Let $A$ be the adjacency matrix of a normally regular digraph. If $\mu=\lambda$ then $A$ is incidence matrix of symmetric 2-design. If $\mu=\lambda+1$ then $A+I$ is incidence matrix of a symmetric 2 -design. In this paper we will often assume that $\mu \notin\{\lambda, \lambda+1\}$.

A tournament is a digraph with the property that for any two distinct vertices $x$ and $y$ exactly one of the edges $x \rightarrow y$ or $y \rightarrow x$ is present.

We will need the following property of regular tournaments in Section 4.

Lemma 3 (Rowlinson [31]). A tournament is normal if and only if it is regular.

Proof. If $A$ is an adjacency matrix of a regular tournament, i.e., $A J=J A=k J$, for some number $k$ then, since $A^{\mathrm{t}}=J-I-A, A A^{\mathrm{t}}=A J-A-A^{2}=J A-A-A^{2}=A^{\mathrm{t}} A$.

Conversely, in a normal digraph every vertex has equal in-degree and out-degree, and in a tournament this degree would be $\frac{v-1}{2}$, where $v$ is the number of vertices.

Example 1. The Cayley graph $\operatorname{Cay}\left(\mathbb{Z}_{5},\{1,2\}\right)$ is a regular tournament and it is therefore normal. But it is not a normally regular digraph.

If a tournament is a normally regular digraph then it is called a doubly regular tournament. It satisfies $k=2 \lambda+1 . \mu$ is arbitrary. Sometimes it is convenient to take $\mu=\lambda$. In other cases we may take $\mu=0$. Such tournaments are also called homogenous tournaments by Kotzig [24], and Ito [16] used the term Hadamard tournaments, as these tournaments are equivalent to skew Hadamard matrices of order $v+1$ (see Reid and Brown [29]).

Thus it is possible that doubly regular tournaments of order $v$ exists for all $v \equiv 3$ $\bmod 4$.

The most important construction of a doubly regular tournament is the Paley tournament which is constructed as follows. Let $\mathbb{F}$ be the field of $q$ elements, $q \equiv 3 \bmod 4$, and let $Q$ be the non-zero squares in $\mathbb{F}$. Then the Cayley graph $\operatorname{Cay}(\mathbb{F}, Q)$ is a doubly regular tournament.

We conclude this section with two small asymmetric normally regular digraphs. 
Example 2. Let $Q=\{1, i, j, k,-1,-i,-j,-k\}$ be the quaternion group. Then the Cayley graph $\operatorname{Cay}(Q,\{i, j, k\})$ is an $N R D(8,3,1,0)$ with the following adjacency matrix

$$
\left(\begin{array}{llllllll}
0 & 1 & 1 & 1 & 0 & 0 & 0 & 0 \\
0 & 0 & 0 & 1 & 1 & 0 & 1 & 0 \\
0 & 1 & 0 & 0 & 1 & 0 & 0 & 1 \\
0 & 0 & 1 & 0 & 1 & 1 & 0 & 0 \\
0 & 0 & 0 & 0 & 0 & 1 & 1 & 1 \\
1 & 0 & 1 & 0 & 0 & 0 & 0 & 1 \\
1 & 0 & 0 & 1 & 0 & 1 & 0 & 0 \\
1 & 1 & 0 & 0 & 0 & 0 & 1 & 0
\end{array}\right)
$$

This is the smallest non-trivial normally regular digraph with $\mu=0$. Normally regular digraphs with $\mu=0$ or $\mu=k$ are characterized in Section 4.

Example 3. $\operatorname{Cay}\left(\mathbb{Z}_{19},\{1,4,6,7,9,11\}\right)$ is an $N R D(19,6,1,3)$. This is the smallest normally regular digraph with $\mu \notin\{k, 0, \lambda, \lambda+1\}$. It belongs to an infinite family constructed in Theorem 34. This digraph is asymmetric and in fact $\lambda_{2}=2 \lambda-\mu$ is negative.

\section{Complementary graphs and the parameters}

The complement of a graph with adjacency matrix $A$ is the graph with adjacency matrix $J-I-A$. The following theorem is proved by an easy computation.

Theorem 4. Let $A$ the adjacency matrix of an $N R D(v, k, \lambda, \mu)$. Then $J-I-A$ is the adjacency matrix of a normally regular digraph with parameters

$$
(\bar{v}, \bar{k}, \bar{\lambda}, \bar{\mu})=(v, v-k-1, v-2 k+\lambda-1, v-2 k+2 \lambda-\mu) .
$$

We will now consider upper and lower bounds on the parameters $\mu, \lambda$ and $\lambda_{2}$. There exist normally regular digraphs for which $\lambda_{2}=2 \lambda-\mu<0$. But in that case there can not be any undirected edges and so the digraph is asymmetric. Note that Theorem 4 is still valid in this case.

Lemma 5. The paramaters of an asymmetric normally regular digraph with $k \geqslant 1$ satisfy the following restriction:

$$
k \geqslant 2 \lambda+1
$$

Proof. The number of edges in the subgraph spanned by the set $x^{+}$of out-neighbours of a vertex $x$ is $k \lambda \leqslant\left(\begin{array}{l}k \\ 2\end{array}\right)$. Thus $2 \lambda \leqslant k-1$.

Lemma 6. The parameters of a normally regular digraph satisfy the inequality

$$
2 k \lambda+(v-2 k-1) \mu \geqslant k^{2}-k .
$$


Proof. Consider the number of triples $(x, y, z)$ of vertices such that $x \rightarrow y \leftarrow z$. We first count the triples by choosing first $(x, z)$ and then $y$ can be chosen in $k, 2 \lambda-\mu, \lambda$ or $\mu$ ways. The number of pairs $(x, z)$ with $x=z$ is $v$. If the graph has $\ell$ undirected edges then the number of pairs $(x, z)$ with $x \leftrightarrow z$ is $2 \ell$, the number of pairs with $x \rightarrow z$ but $x \nLeftarrow z$ is $v k-2 \ell$, the number of pairs with $x \leftarrow z$ but $x \not \rightarrow z$ is $v k-2 \ell$, and the number of non-adjacent pairs is $v^{2}-v-2 v k+2 \ell$. And then the number of triples is

$$
v k+2 \ell(2 \lambda-\mu)+2(v k-2 \ell) \lambda+\left(v^{2}-v-2 v k+2 \ell\right) \mu=v(k+2 k \lambda+(v-2 k-1) \mu) .
$$

If we first choose $y$ and then choose $x$ and $z$ among its in-neighbours then we can express the number of triples as $\sum_{y} d^{-}(y)^{2}$. We do not yet know what the in-degrees are, but we know that the average in-degree is $k$. Therefore $\sum_{y} d^{-}(y)^{2} \geqslant \sum_{y} k^{2}=v k^{2}$. The inequality follows from this.

In the next section we will prove that every vertex has in-degree exactly $k$, and therefore we get equality in Lemma 6.

If a normally regular digraph is a tournament then $\mu$ can be chosen arbitrarily and if it is a complete undirected graph $K_{v}$ then $\mu$ and $\lambda$ can be chosen arbitrarily such that $\lambda_{2}=2 \lambda-\mu=v-2$. In all other cases we have $0 \leqslant \mu \leqslant k$.

Proposition 7. Suppose there exists an $N R D(v, k, \lambda, \mu)$ which is not a tournament or a complete graph. Then

$$
0 \leqslant \mu \leqslant k
$$

and

$$
\lambda_{2}=2 \lambda-\mu \leqslant k-1 .
$$

It can be proved that there is equality in the inequality $\lambda_{2} \leqslant k-1$ if and only if $\mu=0$.

Proof. Suppose that $\mu<0$. Then the digraph does not have any pair of non-adjacent vertices. Since it is not a complete graph, $v-2 k-1>-k$. As $\mu$ is negative we then have $(v-2 k-1) \mu<-k \mu$. As the digraph is not a tournament, there exist undirected edges and $2 \lambda-\mu=\lambda_{2} \leqslant k-1$. From Lemma 6 we have

$$
k^{2}-k \leqslant 2 k \lambda+(v-2 k-1) \mu<2 k \lambda-k \mu \leqslant k(k-1),
$$

a contradiction. Thus $\mu \geqslant 0$.

If $x \leftrightarrow y$ then $x$ and $y$ have $\lambda_{2} \leqslant k-1$ common out-neighbours. So suppose that the digraph is asymmetric. Then by Lemma $5,2 \lambda \leqslant k-1$ and so $2 \lambda-\mu \leqslant k-1$.

Suppose now that $\mu>k$. Let $(v, \bar{k}, \bar{\lambda}, \bar{\mu})$ be the parameters of the complementary normally regular digraph. Then by Theorem 4

$$
v-2 \bar{k}+2 \bar{\lambda}-\bar{\mu}=\mu>k=v-\bar{k}-1,
$$

and so $2 \bar{\lambda}-\bar{\mu}>\bar{k}-1$, a contradiction.

It is convenient to introduce two further parameters of a normally regular digraph:

$$
\eta=k-\mu+(\mu-\lambda)^{2}
$$


and

$$
\rho=k+\mu-\lambda \text {. }
$$

The parameter $\eta$ will play a role similar to that of the order of a symmetric design. Ma [27] uses the parameter $\Delta=4 \eta$ in the study of strongly regular graphs. The factor 4 is necessary in order get an integer for a general strongly regular graph.

Note that it follows from Theorem 4 that the complement of a normally regular digraph $G$ has the same value of $\eta$ as $G$ :

$$
\bar{\eta}=\bar{k}-\bar{\mu}+(\bar{\mu}-\bar{\lambda})^{2}=\eta
$$

\section{Matrix equations}

Using equation 3, the matrix equation in Proposition 1 is equivalent the to following equation.

$$
(A+(\mu-\lambda) I)(A+(\mu-\lambda) I)^{\mathrm{t}}=\eta I+\mu J .
$$

Thus for $B=(A+(\mu-\lambda) I)$ we have

$$
B B^{\mathrm{t}}=\eta I+\mu J
$$

and since $A J=k J$ (every vertex has out-degree $k$ ),

$$
B J=\rho J
$$

We will now prove that a normally regular digraph is normal. The following lemma is a generalization of a proof of the fact that the dual of a symmetric 2-design is also a 2-design, see [2].

Lemma 8. Suppose that $B$ is a non-singular $v \times v$ matrix such that $B B^{t}=\eta I+\mu J$ and $B J=\rho J$ for some constants $\rho, \eta, \mu$. Then $B$ is normal and $\mu v=\rho^{2}-\eta$.

Proof. From $B J=\rho J$ we get $\rho^{-1} J=B^{-1} J$ and

$$
B^{\mathrm{t}}=B^{-1}\left(B B^{\mathrm{t}}\right)=B^{-1}(\eta I+\mu J)=\eta B^{-1}+\mu \rho^{-1} J
$$

Using that $J$ is symmetric, we get from this

$$
\rho J=(B J)^{\mathrm{t}}=J B^{\mathrm{t}}=\eta J B^{-1}+\mu \rho^{-1} J^{2}=\eta J B^{-1}+\mu \rho^{-1} v J .
$$

This implies that

$$
J B^{-1}=\frac{\rho-\mu \rho^{-1} v}{\eta} J
$$

and so

$$
v J=J^{2}=\left(J B^{-1}\right)(B J)=\frac{\rho-\mu \rho^{-1} v}{\eta} \rho v J .
$$


Thus

$$
\frac{\rho-\mu \rho^{-1} v}{\eta}=\rho^{-1}
$$

and $J B^{-1}=\rho^{-1} J$ or $\rho J=J B$. Now equation 5 implies

$$
B^{\mathrm{t}} B=\eta I+\mu \rho^{-1} J B=\eta I+\mu J=B B^{\mathrm{t}} .
$$

Rewriting equation 6 we get $\mu v=\rho^{2}-\eta$.

Corollary 9. The parameters of a normally regular digraph satisfy

$$
\mu v=\rho^{2}-\eta
$$

Theorem 10. Every normally regular digraph is normal.

Proof. Let $A$ be the adjacency of a normally regular digraph and let $B=A+(\mu-\lambda) I$. Then $B B^{\mathrm{t}}=\eta I+\mu J$. Suppose first that $B$ is singular. Then one of the eigenvalues of $\eta I+\mu J$ is zero: $\eta=0$ or $\eta+\mu v=0$. Since $\mu, v \geqslant 0$ this is possible only when $\eta=k-\mu+(\mu-\lambda)^{2}$ is 0 . As $k+(\mu-\lambda)^{2} \geqslant k \geqslant \mu, \mu=k+(\mu-\lambda)^{2}$ implies $k=\mu=\lambda$. This implies that $k=0$. Since a graph with no edges is normal, we may thus assume that $B$ is non-singular, and the result follows from the lemma.

In a normal digraph the number of common in-neighbours of two vertices is equal to the number of common out-neighbors. We therefore get the following.

Corollary 11. An $N R D(v, k, \lambda, \mu)$ is regular of degree $k$, i.e., every vertex has in-degree $k$ and out-degree $k$. Furthermore, the number of common in-neighbours of distinct vertices $x$ and $y$ is

$$
\begin{cases}\mu & \text { if } x \text { and } y \text { are non-adjacent, } \\ \lambda & \text { if either } x \rightarrow y \text { or } y \rightarrow x, \text { but not both, } \\ 2 \lambda-\mu & \text { if } x \leftrightarrow y .\end{cases}
$$

Since we now know that every vertex has in-degree $k$, it follows that there is equality in Lemma 6. Thus

$$
2 k \lambda+(v-2 k-1) \mu=k^{2}-k
$$

This equation is equivalent to equation 7 .

From the theory of symmetric 2-designs we also have the Bruck-Ryser type condition. It is based on the following general lemma from Beth, Jungnickel and Lenz [2]

Lemma 12. Suppose that $N$ is a rational $v \times v$ matrix satisfying the equation

$$
N N^{t}=(a-b) I+b J
$$

for some integers $a>b$ and $v$ odd. Then the equation

$$
x^{2}=(a-b) y^{2}+(-1)^{(v-1) / 2} b z^{2}
$$

has a solution $(x, y, z) \in \mathbb{Z}^{3} \backslash\{(0,0,0)\}$. 
For normally regular digraphs we have the following.

Theorem 13. Suppose that there exist an $N R D(v, k, \lambda, \mu)$.

- If $v$ is even then $\eta=k-\mu+(\mu-\lambda)^{2}$ is a square.

- If $v \equiv 1(\bmod 4)$ then the Diophantine equation $x^{2}-\mu y^{2}=\eta z^{2}$ has an integer solution such that $x, y$, and $z$ are not all zero.

- If $v \equiv 3(\bmod 4)$ then the Diophantine equation $x^{2}+\mu y^{2}=\eta z^{2}$ has an integer solution such that $x, y$, and $z$ are not all zero.

Proof It follows from equation 4 that the determinant of $\eta I+\mu J$ is a square. The eigenvalues of this matrix are $\eta+\mu v=\rho^{2}$ with multiplicity 1 and $\eta$ with multiplicity $v-1$. For the equality we used equation 7 . Thus the result follows when $v$ is even.

For $v$ odd, the theorem follows from equation 4 and the above lemma.

\section{$4 \mu=0$ or $\mu=k$}

In this section we will characterize the structure of normally regular digraphs with $\mu=0$ or $\mu=k$. We first prove that these two cases are complementary. After this we will characterize normally regular digraphs with $\mu=0$ and then get the case $\mu=k$ as a corollary.

Proposition 14. A normally regular digraph satisfies $\mu=k$ if and only if the complementary normally regular digraph satisfies $\mu=0$.

Proof. If $\mu=0$ then it follows from equation 8 that $2 \lambda=k-1$. And then $\bar{\mu}=v-2 k+$ $2 \lambda-\mu=v-k-1=\bar{k}$. If $\mu=k$ then it follows from equation 8 that $v=3 k-2 \lambda$. Thus $\bar{\mu}=v-2 k+2 \lambda-\mu=0$.

\section{$4.1 \mu=0$}

We will first characterize asymmetric normally regular digraphs with $\mu=0$ and then generalize to digraphs with undirected edges.

A normally regular digraph with $\mu=0$ need not be connected. However, each connected component will be a normally regular digraph with the same value of $k$ and $\lambda$. Thus we will only consider normally regular digraphs whose underlying undirected graph is connected. As each vertex has equal in- and out-degree this implies that the digraph is strongly connected. Thus there is a directed path from any vertex to any other vertex. A normally regular digraph with $\mu=0$ may be a doubly regular tournament. Another possibility is that $k=1$ and the digraph is a directed cycle. 
Let $T$ be a tournament with adjacency $A$. Then $\mathcal{D}(T)$ denotes the digraph with adjacency matrix

$$
\left(\begin{array}{cccccccc}
0 & 1 & \ldots & 1 & 0 & 0 & \ldots & 0 \\
0 & & & 1 & & & \\
\vdots & & A & & \vdots & & A^{\mathrm{t}} & \\
0 & & & 1 & & & \\
0 & 0 & \ldots & 0 & 0 & 1 & \ldots & 1 \\
1 & & & 0 & & & \\
\vdots & & A^{\mathrm{t}} & & \vdots & & A & \\
1 & & & & 0 & &
\end{array}\right) .
$$

Thus if $T$ is a tournament with only one vertex then $\mathcal{D}(T)$ is a directed cycle of length 4 . In this section we consider a tournament with one vertex to be doubly regular.

Theorem 15. A connected digraph is an asymmetric normally regular digraph with $\mu=0$ if and only if either

1. it is a directed cycle of length at least 5

2. it is a doubly regular tournament or

3. it is isomorphic to $\mathcal{D}(T)$ for some doubly regular tournament $T$.

Proof. Suppose that $G$ is a connected asymmetric normally regular digraph with $\mu=0$, $k \geqslant 2$ and that $G$ is not a tournament.

As $\mu=0$ we get from equation 8 that $\lambda=\frac{k-1}{2}$. Let $x$ be a vertex of $G$. Then every vertex in $x^{+}$has out-degree $\lambda$ in this subgraph and thus in-degree at most $k-1-\lambda=\lambda$. It follows that $x^{+}$is a regular tournament. Similarly, $x^{-}$is a regular tournament.

Since $G$ is strongly connected and it is not a tournament, there exist a vertex $y \in G$ such that $x$ and $y$ are non-adjacent and there is a path from $x$ to $y$ in $G$. We may choose $y$ such that the (directed) distance from $x$ to $y$ is minimal, i. e. $y$ is dominated by a vertex in $x^{+}$or in $x^{-}$. Since $x$ and $y$ are non-adjacent and $\mu=0, y$ is not dominated by any vertex in $x^{-}$, and similarly $y$ does not dominate any vertex in $x^{+}$. Thus $y$ is dominated by a vertex, say $v$, in $x^{+}$. Suppose there is a vertex $w$ in $x^{+}$that does not dominate $y$. Since $x^{+}$is a regular tournament it is strongly connected, so there is a directed path from $v$ to $w$ in $x^{+}$. On this path there are vertices $u$ and $u^{\prime}$ such that $u \rightarrow u^{\prime}, u \rightarrow y$ but $u^{\prime}$ does not dominate $y$. This is a contradiction to $\mu=0$. Thus every vertex in $x^{+}$ dominates $y$. If another vertex $y^{\prime}$, non-adjacent to $x$ was dominated by a vertex in $x^{+}$, it would be dominated by every vertex in $x^{+}$and so $y$ and $y^{\prime}$ have $k$ common in-neighbours, a contradiction. Thus every vertex in $x^{+}$dominates $\lambda$ vertices in $x^{-}$.

Now a vertex in $x^{-}$dominated by a vertex in $x^{+}$(which dominates $y$ ) must be adjacent to $y$, as $\mu=0$. As above, $y$ then dominates every vertex of $x^{-}$, and every vertex in $x^{-}$ is dominated by $\lambda$ vertices in $x^{+}$. Also every vertex in $x^{-}$dominates exactly $\lambda$ vertices in $x^{+}$. Thus $V(G)=\{x, y\} \cup x^{+} \cup x^{-}$. Furthermore there is an enumeration of vertices 
$x^{+}=\left\{v_{1}, \ldots, v_{n}\right\}$ and $x^{-}=\left\{v_{1}^{\prime}, \ldots, v_{n}^{\prime}\right\}$ such that $v_{i}^{\prime}$ is the unique vertex non-adjacent to $v_{i}$ and vice versa.

If $v_{i} \rightarrow v_{j}$ then, since no vertex dominates both $v_{j}$ and $v_{j}^{\prime}, v_{j}^{\prime} \rightarrow v_{i}$. Similarly $v_{j} \rightarrow v_{i}^{\prime}$ and $v_{i}^{\prime} \rightarrow v_{j}^{\prime}$. Thus the mapping $v_{i} \mapsto v_{i}^{\prime}$ is an isomorphism.

We also see that $v_{\ell}^{\prime}$ is a common out-neighbour of $v_{i}$ and $v_{j}$ if and only if $v_{\ell}$ is a common in-neighbour of $v_{i}$ and $v_{j}$. Thus the number of vertices in $x^{+}$dominating $v_{i}$ and $v_{j}$ plus the number of vertices in $x^{+}$dominated by $v_{i}$ and $v_{j}$ is $\lambda-1$. But since $x^{+}$is a regular tournament it is normal (by Lemma 3) and thus these two numbers are both equal to $\frac{\lambda-1}{2}$ and so $\lambda$ is odd, and $x^{+}$is a doubly regular tournament, $\operatorname{NRD}\left(k, \lambda, \frac{\lambda-1}{2}, \cdot\right)$.

If on the other hand $G$ is a doubly regular tournament with degree $k=2 \lambda+1$ and with vertex-set $\left\{x_{1}, \ldots, x_{n}\right\}, n=2 k+1$, then we may construct a graph with vertex-set $\left\{v_{0}, \ldots, v_{n}, v_{0}^{\prime}, \ldots, v_{n}^{\prime}\right\}$ and edges

$$
v_{0} \rightarrow v_{i} \rightarrow v_{0}^{\prime} \rightarrow v_{i}^{\prime} \rightarrow v_{0}, \text { for } 1 \leqslant i \leqslant n
$$

and

$$
v_{i} \rightarrow v_{j} \rightarrow v_{i}^{\prime} \rightarrow v_{j}^{\prime} \rightarrow v_{i} \text { if } x_{i} \rightarrow x_{j} \text { in } G \text {, for } 1 \leqslant i, j \leqslant n .
$$

It is easy to verify that this new graph is an $\operatorname{NRD}(2 n+2, n, k, 0)$.

The smallest non-trivial example of the type of normally regular digraphs mentioned as possibility 3 is a Cayley graph of the quaternion group of order 8 (see Example 2). In [17] and [23], it is investigated when normally regular digraphs of this type are Cayley graphs or vertex transitive.

We will now characterize normally regular digraphs with $\mu=0$ and with undirected edges. We need a definition to describe the digraphs. Let $G$ be a digraph with vertices $x_{1}, \ldots, x_{n}$. The $s$-clique extension of $G$, denoted by $\mathcal{K}_{s}(G)$ is the digraph with vertex set partitioned in sets $V_{1}, \ldots, V_{n}$ of size $s$ where each $V_{i}$ induce an complete undirected graph and furthermore for $y \in V_{i}$ and $z \in V_{j}, y \rightarrow z$ if and only if $x_{i} \rightarrow x_{j}$ in $G$. If $B$ is an adjacency matrix of $G$ then an adjacency matrix of $\mathcal{K}_{s}(G)$ can be expressed using Kronecker products of matrices (see Hall [10]) as follows $B \otimes J_{s}+I_{n} \otimes\left(J_{s}-I_{s}\right)=$ $(B+I) \otimes J_{s}-I_{n s}$.

Theorem 16. If $G$ is an asymmetric $N R D(v, k, \lambda, 0)$ then for any positive integer $s$, $\mathcal{K}_{s}(G)$ is an $N R D(s v, s k+s-1, s \lambda+s-1,0)$.

Conversely, if $G$ is a connected $N R D(v, k, \lambda, 0)$, then for some number $s$ there is an asymmetric normally regular digraph $G^{\prime}$ with parameters $\left(\frac{v}{s}, \frac{k-s+1}{s}, \frac{\lambda-s+1}{s}, 0\right)$ such that $G$ is isomorphic to $\mathcal{K}_{s}\left(G^{\prime}\right)$.

Proof. Consider a connected normally regular digraph with $\mu=0$. We have that $k=$ $2 \lambda+1$. Then the number of common out-neighbours of $x$ and $y$, where $x \leftrightarrow y$, is $2 \lambda-\mu=k-1$.

Thus if $x \leftrightarrow y$ then $x$ and $y$ have exactly the same set of out-neighbours (and the same set of in-neighbours) other than $y$ and $x$. In particular, if $x \leftrightarrow y \leftrightarrow z$ then $x \leftrightarrow z$. 
It follows that the vertex set is partitioned in sets $V_{1}, \ldots, V_{m}$, such that each $V_{i}$ spans a complete subgraph and there are no undirected edges joining $V_{i}$ and $V_{j}$ for $i \neq j$. If $x \rightarrow y$ for some $x \in V_{i}$ and $y \in V_{j}$ then $x \rightarrow y$ for every $x \in V_{i}$ and $y \in V_{j}$.

Choose $i$ such that $\left|V_{i}\right| \geqslant\left|V_{j}\right|$, for all $j$ and let $s=\left|V_{i}\right|$. Let $V_{i}^{+}$denote the set of out-neighbours outside $V_{i}$ of vertices in $V_{i}$. Then $V_{i}^{+}=V_{i_{1}} \cup \ldots \cup V_{i_{\ell}}$ for some $i_{1}, \ldots, i_{\ell}$ and $V_{i}^{+}$has size $k-(s-1)=2 \lambda+2-s$. In the subgraph spanned by $V_{i}^{+}$every vertex has outdegree $\lambda$. The average in-degree is also $\lambda$. Thus the average number of undirected edges incident with a vertex is at least $2 \lambda-(2 \lambda+2-s-1)=s-1$. By the maximality of $\left|V_{i}\right|=s$, no vertex is incident with more than $s-1$ undirected edges and so $\left|V_{i_{1}}\right|=\ldots=\left|V_{i_{\ell}}\right|=s$. Since the graph is connected, repeated use of this argument shows that $\left|V_{1}\right|=\ldots=\left|V_{m}\right|$. Consider a graph $G^{\prime}$ with vertices $x_{1}, \ldots, x_{m}$ and edges $x_{i} \rightarrow x_{j}$ if $V_{i} \rightarrow V_{j}$. Then $G^{\prime}$ is an $\operatorname{NRD}\left(\frac{v}{s}, \frac{k-s+1}{s}, \frac{\lambda-s+1}{s}, 0\right)$, and $G$ is isomorphic to $\mathcal{K}_{s}\left(G^{\prime}\right)$.

Example 4. If $G^{\prime}$ in this proof is an $N R D(8 t+8,4 t+3,2 t+1,0)$ then $G$ is an $N R D((8 t+$ $8) s,(4 t+4) s-1,(2 t+2) s-1,0)$. Thus an $N R D(16,7,3,0)$ may appear with $(s, t)=(2,0)$ or with $(s, t)=(1,1)$ and so $s$ can not be determined from the parameters.

\section{$4.2 \mu=k$}

Theorem 17. A digraph $G$ is an asymmetric normally regular digraph with $\mu=k$ if and only if there is a number s such that $G$ is obtained from a doubly-regular tournament by replacing each vertex $x$ by a set $V_{x}$ of $s$ new vertices such that if $x \rightarrow y$ in the tournament then $u \rightarrow w$ for every $u \in V_{x}$ and $w \in V_{y}$. Then $s=k-2 \lambda=v-2 k$.

In other words a graph is an asymmetric normally regular digraph with $\mu=k$ if and only if it has an adjacency matrix which is the Kronecker product of an adjacency matrix of a doubly regular tournament and $J_{s}$

Proof. If $G$ is an asymmetric normally regular digraph with $\mu=k$ then the complement $\bar{G}$ of $G$ is a connected normally regular digraph with $\mu=0$ and with no pair of non-adjacent vertices. Then $\bar{G}$ is constructed as in Theorem 16 from an asymmetric normally regular digraph with $\mu=0$ and with no pair of non-adjacent vertices. By Theorem 15 this is a doubly regular tournament.

\section{$5 \quad$ Eigenvalues}

We first state some properties of the spectrum of a normal matrix.

Lemma 18. Let $A$ be a normal matrix with real entries.

Suppose that $\theta$ is an eigenvalue of $A$ of multiplicity $m$. Then

- $\bar{\theta}$ is an eigenvalue of $A^{t}$ of multiplicity $m$.

- $\bar{\theta}$ is an eigenvalue of $A$ of multiplicity $m$. 
- $\theta+\bar{\theta}$ is an eigenvalue of $A+A^{t}$. The multiplicity of this eigenvalue is $m$ if $\theta$ is real, and $2 m$ if $\theta$ is not real.

Conversely, suppose that $\tau$ is an eigenvalue of $A+A^{t}$ of multiplicity $d$.

Then there is an eigenvalue $\theta$ of $A$ such that $\tau=\theta+\bar{\theta}$. If $\theta$ is real then $\theta=\frac{\tau}{2}$ has multiplicity $d$ and if $\theta$ is not real then $\theta$ has multiplicity $\frac{d}{2}$.

Proof. As $A$ is a normal matrix we have the following spectral decomposition

$$
A=\sum_{\theta} \theta E_{\theta}
$$

where the sum is over the eigenvalues of $A$, and $E_{\theta}$ is the matrix of the orthogonal projection on the corresponding eigenspace. As $A$ is real, the adjoint matrix (i.e., the complex conjugate of the transposed matrix) is $A^{\mathrm{t}}$ and since orthogonal projections are self-adjoint we get

$$
A^{\mathrm{t}}=\sum_{\theta} \bar{\theta} E_{\theta}
$$

In particular, $x \in \mathbb{C}^{v}$ is an eigenvector of $A$ with eigenvalue $\theta$ if and only $x$ is an eigenvector of $A^{\mathrm{t}}$ with eigenvalue $\bar{\theta}$.

As the characteristic polynomial of $A$ is real, $\theta$ and $\bar{\theta}$ are roots of equal multiplicity. Thus $\bar{\theta}$ is also an eigenvalue of $A$ of multiplicity $m$. Any eigenvector of $A$ with eigenvalue $\theta$ or $\bar{\theta}$ is an eigenvector of $A+A^{\mathrm{t}}$ with eigenvalue $\theta+\bar{\theta}$. Thus, if $\theta \neq \bar{\theta}$ then $\theta+\bar{\theta}$ is an eigenvalue of $A+A^{\mathrm{t}}$ with multiplicity $2 m$, but if $\theta$ is real then the multiplicity is $m$.

Conversely, as the spectral decomposition of $A+A^{\mathrm{t}}$ can be obtained from the spectral decomposition of $A$, any eigenvalue $\tau$ of $A+A^{\mathrm{t}}$ has the form $\theta+\bar{\theta}$, where $\theta$ is an eigenvalue of $A$.

In general it is not possible to compute the eigenvalues of a normally regular digraph from its parameters. We only know that the degree $k$ is an eigenvalue and (if the graph is connected then) it has multiplicity 1 . We now show that all other eigenvalues lie on a circle in the complex plane with centre $\lambda-\mu$ and radius $\sqrt{\eta}$.

Theorem 19. Suppose that $\theta \neq k$ is an eigenvalue of an $N R D(v, k, \lambda, \mu)$.

Then

$$
|\theta-(\lambda-\mu)|=\sqrt{\eta} .
$$

Proof. Let $A$ be the adjacency matrix of an $\operatorname{NRD}(v, k, \lambda, \mu)$. Let $x \in \mathbb{C}^{v}$ be an eigenvector for $A$ with eigenvalue $\theta$. Then $x$ is eigenvector for $A^{\mathrm{t}}$ with eigenvalue $\bar{\theta}$.

Thus $(\theta+\mu-\lambda)(\bar{\theta}+\mu-\lambda)$ is an eigenvalue of $(A+(\mu-\lambda) I)(A+(\mu-\lambda) I)^{\mathrm{t}}=\eta I+\mu J$. If $\theta \neq k$ then

$$
(\theta+\mu-\lambda)(\bar{\theta}+\mu-\lambda)=\eta
$$

i.e.,

$$
|\theta-(\lambda-\mu)|=\sqrt{\eta}
$$


We now show that equation 9 characterizes normally regular digraphs. This theorem generalizes the well-known result that a connected regular undirected graph with exactly three eigenvalues is strongly regular, see [7].

Theorem 20. Suppose that $G$ is a connected $k$-regular directed graph with a normal adjacency matrix $A$, and suppose there exist real numbers a and $b$ such that every eigenvalue $\theta \neq k$ satisfies $|\theta-a|=b$.

Then either

- $G$ is a normally regular digraph with $\lambda=a+\frac{(k-a)^{2}-b^{2}}{v}$ and $\mu=\frac{(k-a)^{2}-b^{2}}{v}$, where $v$ is the number of vertices, or

- $G$ is a strongly regular graph.

Proof. We can write $A=\sum_{i=1}^{m} \theta_{i} E_{i}$ where $\theta_{1}, \ldots, \theta_{m}$ are the eigenvalues of $A$ and $E_{1}, \ldots, E_{m}$ are the orthogonal projections on the corresponding eigenspaces. We may assume that $\theta_{1}=k$ so that $E_{1}=\frac{1}{v} J_{v}$. Then $A-a I=\sum_{i=1}^{m}\left(\theta_{i}-a\right) E_{i}, A^{\mathrm{t}}-a I=$ $\sum_{i=1}^{m}\left(\overline{\theta_{i}}-a\right) E_{i}$ and so $(A-a I)\left(A^{\mathrm{t}}-a I\right)=\sum_{i=1}^{m}\left(\theta_{i}-a\right)\left(\overline{\theta_{i}}-a\right) E_{i}=(k-a)^{2} E_{1}+b^{2} \sum_{i=2}^{m} E_{i}=$ $(k-a)^{2} \frac{1}{v} J+b^{2}\left(I-\frac{1}{v} J\right)$. This equation is equivalent to $A A^{\mathrm{t}}=\left(b^{2}-a^{2}+\frac{(k-a)^{2}-b^{2}}{v}\right) I+(a+$ $\left.\frac{(k-a)^{2}-b^{2}}{v}\right)\left(A+A^{\mathrm{t}}\right)+\frac{(k-a)^{2}-b^{2}}{v}\left(J-I-A-A^{\mathrm{t}}\right)$. If $G$ is not undirected and not a tournament then clearly, $\lambda$ and $\mu$ are integers and then the theorem follows from Proposition 1. If $G$ is a tournament then $\lambda$ is an integer, there are infinitely many choices for $(a, b)$, and $\mu$ is arbitrary. If $G$ is undirected then it is strongly regular.

Proposition 21. Suppose that $A$ is the adjacency matrix of a connected $N R D(v, k, \lambda, \mu)$. Then

1. The spectrum of $A$ is completely determined by the spectrum of $A+A^{t}$ and the parameters $(v, k, \lambda, \mu)$.

2. If the digraph is not an undirected strongly regular graph then $A$ has at least one non-real eigenvalue.

Proof. 1. Suppose that $\tau$ is an eigenvalue of $A+A^{\mathrm{t}}$ of multiplicity $m$. By Lemma 18, there is an eigenvalue $\theta$ of $A$ such that $\tau=\theta+\bar{\theta}$. The real part of $\theta$ is $\frac{\tau}{2}$. We know from Theorem 19 that $|\theta-(\lambda-\mu)|=\sqrt{\eta}$. Thus $\theta$ is in the intersection of a vertical line determined by the eigenvalue of $A+A^{\mathrm{t}}$ and a circle determined by the parameters.

If this intersection is on the real line then $\left|\frac{\tau}{2}-(\lambda-\mu)\right|=\sqrt{\eta}$ and, by Lemma 18, $\frac{\tau}{2}$ is an eigenvalue of $A$ with multiplicity $m$.

Otherwise, there are two intersections, $\theta$ and $\bar{\theta}$. By Lemma 18, they are both eigenvalues of $A$ with multiplicity $\frac{m}{2}$.

2. If all eigenvalues of $A$ are real then since $A$ is normal it follows that $A$ is selvadjoint and thus symmetric. But the digraph has directed edges.

Remark. If $A$ is the adjacency matrix of a digraph $G$ without undirected edges then $A+A^{\mathrm{t}}$ is the adjacency matrix of the underlying undirected graph of $G$, i.e., the graph obtained by replacing each directed edge by an undirected edge. 
This seems to be all that we can say in general about the the spectrum of a normally regular digraph. But for $\mu=0$ we can at least describe the spectrum for the most important class of normally regular digraphs.

Theorem 22. Suppose that $T$ is a doubly regular tournament such that $G=\mathcal{D}(T)$ is an $N R D(v, k, \lambda, 0)$. Then the eigenvalues of $\mathcal{K}_{s}(G)$ are

$$
s k+s-1, \quad-1, \quad s-1+i s \sqrt{k}, \quad s-1-i s \sqrt{k}
$$

with multiplicities

$$
1, \quad s v-k-2, \quad \lambda+1, \quad \lambda+1 .
$$

Proof. First we consider the eigenvalues of the adjacency matrix $A$ of $G$. Then $A+A^{\mathrm{t}}$ is the adjacency matrix of an imprimitive strongly regular graph. This graph has eigenvalues $2 k, 0$ and -2 with multiplicities $1, k+1$ and $k$. Thus if $\theta \neq k$ is an eigenvalue of $A$ then $\theta+\bar{\theta} \in\{0,-2\}$. By equation 9, $|\theta-\lambda|=\lambda+1$, as $\eta=k-\mu+(\mu-\lambda)^{2}=2 \lambda+1+\lambda^{2}$. If $\theta+\bar{\theta}=-2$ then $\theta=-1$. The multiplicity is $k$. If $\theta+\bar{\theta}=0$ then $\theta= \pm i \sqrt{k}$. These two eigenvalues have multiplicity $\frac{1}{2}(k+1)=\lambda+1$.

Thus $A+I$ has eigenvalues $k+1,0$ and $1 \pm i \sqrt{k}$. The matrix $(A+I) \otimes J_{s}-I_{v s}$ is an adjacency matrix of $\mathcal{K}_{s}(G)$. We first compute the eigenvalues of $(A+I) \otimes J_{s}$ and then subtract 1 .

For each eigenvector $x \in \mathbb{C}^{v}$ of $A+I$ with eigenvalue $\theta$ we can replace each entry $x_{i}$ with $s$ entries equal to $x_{i}$ to get an eigenvector in $\mathbb{C}^{v s}$ of $(A+I) \otimes J_{s}$ with eigenvalue $s \theta$. Furthermore we can get $v(s-1)$ orthogonal eigenvectors with eigenvalue 0 , by taking one of the blocks to be orthogonal to $(1, \ldots, 1)^{\mathrm{t}} \in \mathbb{C}^{s}$ and all other entries 0 .

Note that the two non-isomorphic $\operatorname{NRD}(16,7,3,0)$ mentioned in Example 4 have different spectra.

\section{Relation to association schemes}

An asymmetric normally regular digraph may have the additional property that $A^{2}$ (where $A$ is the adjacency matrix) can be expressed as linear combination of $A, A^{\mathrm{t}}, I$ and $J$. In that case the digraph is related to an association scheme.

Definition 2. Let $X$ be finite set and let $\left\{R_{0}, R_{1}, \ldots, R_{d}\right\}$ be a partition of $X \times X$. Then $\mathcal{X}=\left(X,\left\{R_{0}, R_{1}, \ldots, R_{d}\right\}\right)$ is an association scheme with $d$ classes if the following conditions are satisfied

- $R_{0}=\{(x, x) \mid x \in X\}$,

- for each $i \in\{0, \ldots, d\}$ there exists $i^{\prime} \in\{0, \ldots, d\}$ such that

$$
R_{i^{\prime}}=\left\{(x, y) \mid(y, x) \in R_{i}\right\}
$$


- for each triple $(i, j, k), i, j, k \in\{0, \ldots, d\}$ there exist a number $p_{i j}^{k}$ such that for all $x, y \in X$ with $(x, y) \in R_{k}$ there are exactly $p_{i j}^{k}$ elements $z \in X$ such that $(x, z) \in R_{i}$ and $(z, y) \in R_{j}$.

If $i=i^{\prime}$ for all $i$ then the association scheme is called symmetric, otherwise it is nonsymmetric.

The relation $R_{i}, i=1, \ldots, d$ can be considered as an undirected graph if $i=i^{\prime}$ and as a directed graph if $i \neq i^{\prime}$.

It is well-known that an undirected graph is strongly regular if and only if it is a relation of a symmetric association scheme with two classes. And a directed graph is a doubly regular tournament if and only if it is a relation of a non-symmetric association scheme with two classes.

For a general introduction to association schemes we refer to Bannai and Ito [1]. Goldbach and Classen [8] have studied non-symmetric association schemes with three classes and in [9] they describe the structure of non-symmetric association schemes with three classes that are imprimitive, i.e., at least one the graphs $R_{1}, R_{2}, R_{3}$ is disconnected. For tables of feasible parameter sets see [19]. Association schemes with four classes and with no symmetric relations other than $R_{0}$ have been investigated by Jianmin Ma and Kaishun Wang [26].

Proposition 23. 1. If $\left(X,\left\{R_{0}, R_{1}, R_{2}, R_{3}\right\}\right)$ is an association scheme with $1^{\prime}=2$ then $R_{1}$ and $R_{2}$ are asymmetric normally regular digraphs.

2. If $\left(X,\left\{R_{0}, R_{1}, R_{2}, R_{3}, R_{4}\right\}\right)$ is an association scheme with $1^{\prime}=2$ and $3^{\prime}=4$ then $R_{1}, \ldots, R_{4}$ are asymmetric normally regular digraphs.

Proof. We prove case 2 for the graph $R_{1}$. The other cases are similar. The graph $R_{1}$ is regular with degree $p_{12}^{0}$. Suppose that $x$ and $y$ are adjacent in $R_{1}$. We may assume that $(x, y) \in R_{1}$. Then the number of common out-neighbours of $x$ and $y$ is $p_{12}^{1}$. Suppose now that $x$ and $y$ are non-adjacent. We may assume that $(x, y) \in R_{3}$, since otherwise $(x, y) \in R_{4}$ and then $(y, x) \in R_{3}$. Then the number of common out-neighbours of $x$ and $y$ is $p_{12}^{3}$.

It follows from Proposition 21 that the adjacency matrix of a normally regular digraph has at least three distinct eigenvalues. The normally regular digraphs constructed from non-symmetric association schemes with 2, 3 or 4 classes have 3, 4 and 5 distinct eigenvalues, respectively.

We now consider normally regular digraphs where the number of distinct eigenvalues is either 3, 4 or 5, and try to construct association schemes.

In the following proofs it is easier to work with a reformulation of the definition of association schemes in terms of matrices.

Proposition 24. Suppose that $R_{0}, \ldots, R_{d}$ are relations on a set $X$, with adjacency matrices $A_{0}, \ldots, A_{d}$. Let $\mathcal{A}$ be the vector space spanned by $\left\{A_{0}, \ldots, A_{d}\right\}$. Then $\left(X,\left\{R_{0}, \ldots, R_{d}\right\}\right)$ is an association scheme if and only if $A_{0}+\ldots+A_{d}=J$ and 
- $I \in\left\{A_{0}, \ldots, A_{d}\right\},\left(\operatorname{say} I=A_{0}\right)$,

- $A_{i}^{t} \in\left\{A_{0}, \ldots, A_{d}\right\}$, for all $i$, and

- $\mathcal{A}$ is closed under matrix multiplication.

In fact, $A_{i} A_{j}=\sum_{k} p_{i j}^{k} A_{k}$.

By Theorem 20, it is not necessary to assume that the digraphs considered in the following three results are normally regular. This follows from other properties.

Theorem 25. Suppose that $G$ is a connected $k$-regular digraph with a normal adjacency matrix A having exactly three distinct eigenvalues. Then either

- $G$ is an undirected strongly regular graph or

- $G$ is a doubly regular tournament and therefore a normally regular digraph, and the eigenvalues are $k,-\frac{1}{2}+i \sqrt{\lambda+\frac{3}{4}}$ and $-\frac{1}{2}-i \sqrt{\lambda+\frac{3}{4}}$, with multiplicities $1, k$ and $k$ respectively, where $k=2 \lambda+1$.

In both cases we have an association scheme with 2 classes.

Proof. Let $k, \theta$ and $\tau$ be the eigenvalues and let $E_{k}, E_{\theta}$ and $E_{\tau}$ be the orthogonal projections on the eigenspaces. Then $\mathcal{A}=\operatorname{span}\left\{E_{k}, E_{\theta}, E_{\tau}\right\}$ is closed under multiplication, as the projections are idempotents and the eigenspaces are orthogonal. We have

$$
\begin{gathered}
I=E_{k}+E_{\theta}+E_{\tau}, \\
J=v E_{k} \\
A=k E_{k}+\theta E_{\theta}+\tau E_{\tau},
\end{gathered}
$$

and the adjoint of $A$ is

$$
A^{\mathrm{t}}=k E_{k}+\bar{\theta} E_{\theta}+\bar{\tau} E_{\tau} .
$$

It follows that $I, J, A$ and $A^{\mathrm{t}}$ are linearly dependent, so there exists rational numbers $a, b, c, d$ not all 0 such that

$$
a A+b A^{\mathrm{t}}=c(J-I)+d I .
$$

Clearly $d=0$. If $c=0$ then $A=A^{\mathrm{t}}$. Otherwise either $A+A^{\mathrm{t}}=J-I$ or $A=A^{\mathrm{t}}=J-I$.

Thus either $\mathcal{A}=\operatorname{span}\left\{I, A, A^{\mathrm{t}}\right\}$ or $\mathcal{A}=\operatorname{span}\{I, A, J-I-A\}$ and these matrices satisfy the properties required in Proposition 24.

The spectrum can be computed as described in Proposition 21.

Theorem 26. Suppose that $G$ is a connected asymmetric $k$-regular digraph with a normal adjacency matrix A having exactly four distinct eigenvalues. Then $G$ is a relation of a non-symmetric association scheme with three classes and $G$ is a normally regular digraph. 
Proof. Let $A$ be the adjacency matrix of $G$. Let $\mathcal{A}=\operatorname{span}\left\{I, A, A^{\mathrm{t}}, J-I-A-A^{\mathrm{t}}\right\}$. We need to show that $\mathcal{A}$ is closed under matrix multiplication. Let $k, \tau, \theta$ and $\bar{\theta}$ be the eigenvalues of $A$. Let $E_{k}, E_{\tau}, E_{\theta}$ and $E_{\bar{\theta}}$, respectively, be the orthogonal projections on the corresponding eigenspaces. Then

$$
\begin{gathered}
I=E_{k}+E_{\tau}+E_{\theta}+E_{\bar{\theta}}, \\
J=v E_{k}, \\
A=k E_{k}+\tau E_{\tau}+\theta E_{\theta}+\bar{\theta} E_{\bar{\theta}},
\end{gathered}
$$

and

$$
A^{\mathrm{t}}=k E_{k}+\tau E_{\tau}+\bar{\theta} E_{\theta}+\theta E_{\bar{\theta}} .
$$

Thus $\left\{E_{k}, E_{\tau}, E_{\theta}, E_{\bar{\theta}}\right\}$ is a basis of $\mathcal{A}$. Since these projections are idempotents and the product of distinct projections is $0, \mathcal{A}$ is closed under multiplication.

If an asymmetric normally regular digraph has exactly five distinct eigenvalues then it may have either three real eigenvalues and one pair of complex conjugate eigenvalues or else it has one real eigenvalues and two pairs complex conjugate eigenvalues. In the latter cases it seems likely that the graph is a relation of a non-symmetric association scheme with four classes. We can only prove the following.

Proposition 27. Suppose that $G$ is a connected asymmetric $k$-regular digraph with a normal adjacency matrix having exactly five distinct eigenvalues $k, \theta, \bar{\theta}, \tau, \bar{\tau}$, where $\theta$ and $\tau$ have distinct real parts. Then $G$ is a normally regular digraph and it is an orientation of a strongly regular graph.

Proof. There is a circle in the complex plane passing through $\theta, \bar{\theta}, \tau$ and $\bar{\tau}$ with centre in the intersection of the real axis and the perpendicular bisector of $\theta$ and $\tau$. By Theorem 20, $G$ is normally regular.

The underlying undirected graph is regular and has exactly three distinct eigenvalues $2 k, \theta+\bar{\theta}$ and $\tau+\bar{\tau}$. Thus it is strongly regular, see [7].

Conversely, it follows from Proposition 21 that if a normally regular digraph is an orientation of a strongly regular graph then the number of distinct eigenvalues is either four or five.

\section{Group divisible partitions}

We start with the definition of two types of partitions of the vertex set.

Suppose that the vertex set of a normally regular digraph is partitioned in sets $V_{1}, \ldots, V_{m}$. Then we say that $V_{1}, \ldots, V_{m}$ is an equitable partition if there exists constants $c_{i j}, d_{i j}$ for $i, j \in\{1, \ldots, m\}$ such that for every vertex $x \in V_{i},\left|x^{+} \cap V_{j}\right|=c_{i j}$ and for every vertex $y \in V_{j},\left|y^{-} \cap V_{i}\right|=d_{i j}$. If $\left|V_{i}\right|=\left|V_{j}\right|$ then $c_{i j}=d_{i j}$. We say that $C=\left(c_{i j}\right)_{i, j=1, \ldots, m}$ is the quotient matrix of the equitable partition, see [7]. 
Let $G$ be an asymmetric $\operatorname{NRD}(v, k, \lambda, \mu)$. Then we say that $G$ is group divisible if $G$ is a multipartite tournament, i.e., if $V(G)$ can be partitioned in sets $V_{1}, \ldots, V_{r}$ such that there is an edge between $x \in V_{i}$ and $y \in V_{j}$ if and only if $i \neq j$.

Since $G$ is regular the sets $V_{i}$ all have the same size, say $\left|V_{i}\right|=s=v-2 k$, for $i=1, \ldots, r$. Then $v=r s$ and $k=\frac{1}{2}(r-1) s$. We assume that $s>1$.

The adjacency matrix of a group divisible normally regular digraph with $\mu \neq \lambda$ is also the incidence matrix of a group divisible design, see [2]

Lemma 28. Let $G$ be an asymmetric $N R D(v, k, \lambda, \mu)$ and suppose that $G$ is group divisible with partition $V_{1}, \ldots, V_{r}$. Then $V_{1}, \ldots, V_{r}$ is an equitable partition.

Proof. For $z \in V_{i}$ let $c_{i j}(z)=\left|z^{+} \cap V_{j}\right|$. We need to prove that $c_{i j}(z)$ does not depend on $z$. Let $x \in V_{i}$ and $y \in V_{j}$. We count the vertices in $S=\{z \mid x \rightarrow z \rightarrow y\}$ in two ways. The number of out-neighbours of $x$ outside $V_{j}$ is $k-c_{i j}(x) . \lambda$ of these outneighbours are common out-neighbours of $x$ and $y$. The remaining $k-c_{i j}(x)-\lambda$ vertices are in $S$. Similarly, $y$ has $k-\left(\left|V_{i}\right|-c_{j i}(y)\right)=k-\left(s-c_{j i}(y)\right)$ in-neighbours outside $V_{i}$. $k-\left(s-c_{j i}(y)\right)-\lambda$ vertices are in $S$. Thus $s=c_{i j}(x)+c_{j i}(y)$, for all $x \in V_{i}$.

Proposition 29. Let $A$ be the adjacency matrix of a group divisible normally regular digraph. Then

- A has either 4 or 5 distinct eigenvalues.

- If A has 4 distinct eigenvalues then the graph is a relation of a non-symmetric imprimitive association scheme with three classes.

- If A has 5 distinct eigenvalues then $r$ and $s$ are odd.

Proof. Since $A+A^{\mathrm{t}}$ is a strongly regular graph it has three distinct eigenvalues. Then by Theorem 19 and Proposition 21, $A$ has either 4 or 5 eigenvalues.

It follows from Theorem 26 that if $A$ has 4 eigenvalues then the graph is a relation of a non-symmetric association scheme with three classes.

It follows from Proposition 21 that if $A$ has 5 distinct eigenvalues then eigenvalue $k$ has multiplicity 1 and the other 4 eigenvalues have pairwise the same multiplicity. Thus the number of vertices is odd, and so $r$ and $s$ are odd.

The only known examples of group divisible normally regular digraphs with an odd number of vertices satisfies $\mu=k$ and then by Theorem 17 they are a relation of a non-symmetric imprimitive association scheme with three classes.

Conjecture 1. Any group divisible normally regular digraph is a relation of a nonsymmetric imprimitive association scheme with three classes.

Example 5. The parameters of a group divisible normally regular digraph must satisfy that $v-2 k$ divides $v$. This is satisfied by $(v, k, \lambda, \mu)=(16,6,2,2)$. There are four asymmetric normally regular digraphs with these parameters. Two of these are group divisible and thus are relations of an association scheme. 
One of these is a Cayley graph

$$
\operatorname{Cay}\left(\mathbb{Z}_{4} \times \mathbb{Z}_{4},\{(0,3),(1,3),(2,1),(3,0),(3,2),(3,3)\}\right) .
$$

The independent sets of vertices in this digraph are the cosets of the subgroup $\{(0,0),(0,2)$, $(2,0),(2,2)\}$.

One of the normally regular digraphs with these parameters that is not group divisible has vertex set $\left\{a_{i}, b_{i} \mid i \in \mathbb{Z}_{8}\right\}$ and edges

$$
\begin{gathered}
a_{i} \rightarrow a_{i+1}, a_{i+2}, b_{i}, b_{i+1}, b_{i+4}, b_{i+6}, \quad i \in \mathbb{Z}_{8}, \\
b_{i} \rightarrow b_{i-1}, b_{i-2}, a_{i-2}, a_{i-3}, a_{i-5}, a_{i-7}, \quad i \in \mathbb{Z}_{8} .
\end{gathered}
$$

Thus group divisibility is not determined by the parameters.

\section{Combinatorial results for small $\lambda$}

In this section we use combinatorial methods to prove non-existence for certain parameter sets where $\lambda$ is small. If $\lambda$ is so small that $2 \lambda-\mu$ is negative then only the asymmetric case need to be considered.

Theorem 30. If there exist an asymmetric normally regular digraph with parameters $(v, k, \lambda, \mu)$ where $2 \mu>k+\lambda$ then the graph is group-divisible and $v-2 k$ divides $v$.

Proof. Suppose that $G$ is an $\operatorname{NRD}(v, k, \lambda, \mu)$. Let $x$ be a vertex in $G$. Let $y$ and $z$ be vertices in $V(G)-\{x\}-x^{+}-x^{-}$. Then $x^{+} \cap y^{+}$and $x^{+} \cap z^{+}$each consist of $\mu$ vertices in the set $x^{+}$of $k$ vertices. Thus $\left|y^{+} \cap z^{+}\right| \geqslant 2 \mu-k>\lambda$ and so $y$ and $z$ are nonadjacent. It follows that every vertex in $G$ belongs to a unique independent set of $v-2 k$ vertices and so $G$ is group divisible with $\frac{v}{v-2 k}$ groups.

Corollary 31. Suppose that $2 \lambda-\mu<0,2 \mu>k+\lambda$ and $v-2 k$ does not divide $v$ then an $N R D(v, k, \lambda, \mu)$ does not exist.

Theorem 32. If a normally regular digraph with $\lambda=0, \mu \neq k$ and $\mu \geqslant 2$ exists then $k \geqslant 2 \mu+\frac{1}{2}+\sqrt{2 \mu+\frac{1}{4}}$.

From Theorem 17 we know that an $\operatorname{NRD}(v, k, 0, k)$ is obtained from a directed triangle by replacing each vertex by $k$ vertices.

Proof Suppose that $G$ is an $\operatorname{NRD}(v, k, 0, \mu)$ with $\mu \neq k$. Since $2 \lambda-\mu<0$ any such normally regular digraph is asymmetric. In this proof we use notation $U_{x}=V(G)-\{x\}-$ $x^{+}-x^{-}$for a vertex $x$ in $G$. By equation 8 we have

$$
\left|U_{x}\right|=\frac{k(k-1)}{\mu}
$$

By Proposition $7, k>\mu$.

Claim 1: $k>2 \mu$. 
Proof Let $x$ be any vertex in $G$. By Theorem 30, $U_{x}$ is an independent set if $\mu<k<2 \mu$.

So suppose that $k=2 \mu$ and $k>2$. By equation $10,\left|U_{x}\right|=2 k-2$. Suppose that $y, z \in U_{x}$ and $y$ dominates $z . x^{+} \cap y^{+}$and $x^{+} \cap z^{+}$are disjoint sets (as $\lambda=0$ ) of cardinality $\mu$. Thus their union is $x^{+}$. $z$ has $\mu$ in-neighbours in $x^{-}$, no in-neighbours in $x^{+}$and thus $\mu$ in-neighbours in $U_{x}$. Let $y^{\prime} \in U_{x}$ be another vertex dominating $z$. Then $y^{\prime}$ and $z$ have no common out-neighbours in $x^{+}$, i.e., $x^{+} \cap\left(y^{\prime}\right)^{+}=x^{+} \cap y^{+}$. Thus $y$ and $y^{\prime}$ have at least $\mu+1$ common out-neighbours, a contradiction.

Thus $U_{x}$ is an independent set.

Let $z \in x^{+}$. Every vertex other than $x$ dominating $z$ belongs to $U_{x}$. As $U_{x}$ is independent, no vertex dominates both $z$ and $y \in U_{x}$ and so $z$ is adjacent to every vertex in $U_{x}$ (but to no other vertex in $x^{+}$). Thus $z$ is adjacent to $2 k-1-\left|U_{x}\right|$ vertices in $x^{-}$. By equation 10 and $\mu<k \leqslant 2 \mu$, we have $k>2 k-1-\left|U_{x}\right| \geqslant 1$, and so there is a vertex $w \in x^{-}$adjacent to $z$ and a vertex $u \in x^{-}$not adjacent to $z$. Then $w$ and $z$ are adjacent vertices in $U_{u}$, a contradiction.

Claim 2: $k>2 \mu+1$.

Proof Suppose that $k=2 \mu+1$ and let $x$ be a vertex in $G$. By equation $10,\left|U_{x}\right|=2 k$. Since $G$ is regular and $\left|U_{x} \cup\{x\}\right|>\left|x^{+} \cup x^{-}\right|, U_{x}$ cannot be an independent set. Let $y, z \in U_{x}$ such that $y \rightarrow z$. $y$ and $z$ have no common out-neighbours in $x^{+}$so there a unique vertex $w \in x^{+}$which is not dominated by $y$ or $z$. The in-neighbours of $z$ are $\mu$ vertices in $x^{-}$, possibly $w$, and at least $\mu$ vertices in $U_{x}$. Let $y^{\prime} \neq y$ be a vertex in $U_{x}$ dominating $z$. Since $y^{\prime}$ and $z$ have no common out-neighbours in $x^{+}$and $y$ and $y^{\prime}$ have only $\mu$ common out-neighbours, $y^{\prime}$ dominates $w$. Since $\lambda=0, w$ does not dominate $z$, and so $z$ has $\mu+1$ in-neighbours in $U_{x}$. We have now shown that in the graph spanned by $U_{x}$ any vertex has in-degree either 0 or $\mu+1$ and, by symmetry, it has out-degree either 0 or $\mu+1$. Thus the in-neighbours of $z$ in $U_{x}$ has out-degree $\mu+1$. Any two in-neighbours of $z$ in $U_{x}$ have at least $\mu-1$ common out-neighbours in $\left(x^{+} \cap y^{+}\right) \cup\{w\}$. Thus $z$ is their only common out-neighbour in $U_{x}$. Counting the vertices in $U_{x}$ we have

$$
4 \mu+2=\left|U_{x}\right| \geqslant 1+(\mu+1)+(\mu+1) \mu,
$$

and so $\mu=2$.

Now $\left|U_{x}\right|=10$ and we have at least 7 vertices of in-degree 3 in $U_{x}$ and, by symmetry, at least 7 vertices of out-degree 3 . So there is a vertex with out-degree and in-degree 3 . We may assume that $z$ is such a vertex. Then $z$ dominates a vertex which is also dominated by an in-neighbour of $z$. This is a contradiction to $\lambda=0$. This proves claim 2 .

Let $r=k-2 \mu$. Then $r \geqslant 2$, by Claim 2. By equation $10, \mu$ divides $k(k-1)=$ $(2 \mu+r)(2 \mu+r-1)=\mu(4 \mu+4 r-2)+r(r-1)$. Thus $\mu$ divides $r(r-1)$ and so $r^{2}-r=s \mu$, for some positive integer $s$. Then $r=\frac{1}{2}+\sqrt{s \mu+\frac{1}{4}}$.

If $s=1$ then $\mu=r(r-1)$ and $k=2 \mu+r=r(2 r-1)$. From equation 10, we see that $v=1+2 k+\frac{k(k-1)}{\mu}=2 k+4 r^{2}$ is even and so $\eta$ is a square, by Theorem 13. But $\eta=k-\mu+\mu^{2}=r^{2}\left((r-1)^{2}+1\right)$ cannot be a square. Thus $s \geqslant 2$. This proves the theorem. 


\section{Normally regular digraphs as quotient graphs}

\subsection{Subplane partition}

Fossorier, Ježek, Nation and Pogel [6] considered partition of a projective plane of order $n$ into subplanes $\pi_{1}, \ldots, \pi_{v}$ of order $q, v=\frac{n^{2}+n+1}{q^{2}+q+1}$. They say that such a partition is ordinary if for each pair $(i, j)$ either each point of $\pi_{i}$ is incident with a line of $\pi_{j}$ or no point of $\pi_{i}$ is incident with a line of $\pi_{j}$.

For an ordinary partition of a projective plane they consider the quotient graph with vertices $\pi_{1}, \ldots, \pi_{v}$ and an edge $\pi_{i} \rightarrow \pi_{j}$ if the points of $\pi_{i}$ are incident with lines of $\pi_{j}$. They proved that this quotient graph is what they called an ordinary graph. This is a normally regular digraph in our terminology.

Theorem 33 (Fossorier, Ježek, Nation and Pogel [6]). If a projective plane of order $n$ has an ordinary partition into projective planes of order $q$ then the quotient graph is a normally regular digraph with $(v, k, \lambda, \mu)=\left(\frac{n^{2}+n+1}{q^{2}+q+1}, n-q, q^{2}, q^{2}+q+1\right)$.

For a partition into Baer subplanes, i.e., $n=q^{2}$, the quotient graph is a complete undirected graph.

Theorem 36 below describes the special case of this theorem where we consider desarguesian planes. Theorem 34 may also be seen as a special case of Theorem 33 where $q=1$.

\subsection{Bipartite graphs of diameter 3}

Delorme, Jørgensen, Miller and Pineda-Villavicencio [4] considered a similar quotient graph construction. In this paper we considered bipartite $q+1$ regular graphs with diameter 3 and with $2\left(q^{2}+q\right)$ vertices. (The largest possible bipartite $q+1$ regular graph with diameter 3 has $2\left(q^{2}+q+1\right)$ vertices and it appears only as incidence graph of a projective plane of order q.) In such graphs the vertices are partitioned into cycles of length 4 . It is proved that the graph obtained by directing all edges from one bipartition class to the other and then identifying each 4-cycle to a vertex is a normally regular digraph with $(v, k, \lambda, \mu)=\left(\frac{q^{2}+q}{2}, q-1,0,2\right)$.

This was our original motivation for studying normally regular digraphs.

\section{Constructions}

In this section we give a number of constructions of families of normally regular digraphs. Most of these constructions use Cayley graphs of abelian groups.

\subsection{Asymmetric Cayley graph constructions}

The first construction uses a partition of a projective plane into triangles. If a triangle is considered to be a "subplane" of order 1 then this is a special case of the construction in Theorem 33. 
This construction was also found by de Resmini and Jungnickel [30] as an example of what they call a failed symmetric design.

Theorem 34. Let $k$ be a multiple of 3 such that $k+1$ is a prime power. Then there exists $S \subset \mathbb{Z}_{v}, v=\frac{k^{2}+3 k+3}{3}$ such that $\operatorname{Cay}\left(\mathbb{Z}_{v}, S\right)$ is an asymmetric $N R D(v, k, 1,3)$.

Proof When $\lambda=1$ and $\mu=3, \eta=k+1$, which is assumed to be a prime power. By equation $7, v=\frac{\eta^{2}+\eta+1}{3}$.

By Singer's theorem [32] there exist a cyclic planar difference set of order $\eta$, i. e. a subset $D$ of $\mathbb{Z}_{3 v}$ with $|D|=\eta+1$ such that each non-zero element of $\mathbb{Z}_{3 v}$ is a difference of exactly one ordered pair of elements in $D$. In particular there is a unique pair of difference $v$. By adding a constant to $D$ if necessary, we may assume that $v, 2 v \in D$. Let $D^{\prime}=D \backslash\{v, 2 v\}$, and let $S \subseteq \mathbb{Z}_{v}$ be the numbers congruent to numbers in $D^{\prime}$ modulo $v$.

As $v$ is not a difference in $D^{\prime},|S|=\eta-1=k$.

Suppose that $x$ and $-x$ are both in $S$. Then for some $a, b \in\{0,1,2\}, x+a v,-x+b v \in$ $D^{\prime}$. Choose $i, j \in\{1,2\}$ such that $a+b \equiv i+j(\bmod 3)$. Then we have two equal differences in $D$

$$
(x+a v)-i v=j v-(-x+b v)
$$

a contradiction. Thus $S \cap-S=\varnothing$.

If $x \in \mathbb{Z}_{v}$ is congruent $(\bmod v)$ to a difference of elements in $D$, one of which is $v$ or $2 v$, then either $x \equiv a-i v$ or $x \equiv i v-a$ for $a \in D$ and $i \in\{1,2\}$, i. e. $x$ or $-x \in S$. Conversely if $x \in S$ or $-x \in S$ then $x$ is in exactly to ways congruent $(\bmod v)$ to a difference of two elements in $D$ one of which is $v$ or $2 v$.

Let $x \in \mathbb{Z}_{v} \backslash(S \cup-S \cup\{0\})$. Then each of $x, x+v$ and $x+2 v$ can be written in exactly one way as a difference of elements in $D$, in fact in $D^{\prime}$. Thus $x$ can be written in exactly three ways as a difference of elements in $S$.

If $x \in S \cup-S$ then only one of the three pairs of elements in $D$ whose difference is congruent to $x(\bmod v)$ is in $D^{\prime}$. Thus $x$ can be written in exactly one way as a difference of elements in $S$.

Hence $\operatorname{Cay}\left(\mathbb{Z}_{v}, S\right)$ is an $\operatorname{NRD}(v, k, 1,3)$.

In the next theorem we construct a family of Cayley graphs of abelian but not necessarily cyclic groups. It is well-known that this digraph is one of the classes of a (so-called cyclotomic) association scheme with four classes.

Theorem 35. Suppose that $v$ is a prime power, $v \equiv 5 \bmod 8$. Let $D$ denote the following subset of $G F[v]$ :

$$
D=\left\{x^{4} \mid x \neq 0\right\}
$$

Then the Cayley graph of the additive group of GF[v] generated by $D$ is a normally regular digraph with $v=4 k+1=8(\mu+\lambda)+5$.

Proof As $v \equiv 5 \bmod 8$, the set $D$ has cardinality $k=\frac{v-1}{4}$ and $-1 \notin D$. Thus $D \cup-D$ is the set of squares in $G F[v]$. This means that the cosets of the subgroup (of the multiplicative group) $D$ are $D,-D, R$, and $-R$ for some set $R$. Let $D=\left\{1, q_{2}, \ldots, q_{k}\right\}$. Then $q q_{i}-q, 2 \leqslant i \leqslant k, q \in D$ is the set of differences, we want to consider. For a 
fixed $i$, every element in the coset to which $q_{i}-1$ belongs appears exactly once as a difference $q q_{i}-q, q \in D$. This means that if among the differences $q_{2}-1, \ldots, q_{k}-1$, the number of elements in $D,-D, R$ and $-R$, are $\lambda_{1}, \lambda_{2}, \mu_{1}$ and $\mu_{2}$, respectively, then among all differences of distinct element of $D$ an element appears $\lambda_{1}, \lambda_{2}, \mu_{1}$, or $\mu_{2}$ times according to whether it belongs to $D,-D, R$, or $-R$. Since for every $x, x$ and $-x$ appears as a difference the same number of times, $\lambda_{1}=\lambda_{2}$ and $\mu_{1}=\mu_{2}$.

The only known infinite family of primitive non-symmetric association schemes with three classes is a family constructed by Liebler and Mena [25]. For every $s=2^{n}$, they constructed a so-called distance regular digraph of girth 4 and degree $s\left(2 s^{2}-1\right)$, as a Cayley digraph of $\mathbb{Z}_{4} \times \ldots \times \mathbb{Z}_{4}$. Their graph is in fact an $\operatorname{NRD}\left(4 s^{4}, s\left(2 s^{2}-1\right), 2 s(s-1), s(s-1)\right)$.

Some of the normally regular digraphs constructed in the next two subsections are also asymmetric.

\subsection{Construction from desarguesian planes}

We will now consider the subplane partition described in Section 9.1 for desarguesian projective planes.

Theorem 36. Let $q$ be a prime power and let $r \geqslant 2$ be an integer not divisible by 3. Let $v=\frac{q^{2 r}+q^{r}+1}{q^{2}+q+1}$. Then there exists a set $S \subset \mathbb{Z}_{v}$ such that $C a y\left(\mathbb{Z}_{v}, S\right)$ is a normally regular digraph with parameters $\left(v, q^{r}-q, q^{2}, q^{2}+q+1\right)$.

Proof. Let GF $\left[q^{3 r}\right]$ be the field with $q^{3 r}$ elements and with primitive element $\alpha$. Then $\mathrm{GF}\left[q^{r}\right]$ and $\mathrm{GF}\left[q^{3}\right]$ are subfields and their intersection is $\mathrm{GF}[q]$ as 3 does not divide $r$.

Let $\beta=\alpha^{\left(q^{3 r}-1\right) /\left(q^{3}-1\right)}$. Then $\beta$ is a primitive element of $\operatorname{GF}\left[q^{3}\right]$ and $\beta \notin \operatorname{GF}\left[q^{r}\right]$. Thus when $\mathrm{GF}\left[q^{3 r}\right]$ is considered as a 3 dimensional vector space over $\operatorname{GF}\left[q^{r}\right]$ then vectors 1 and $\beta$ span a 2 dimensional subspace $U$. Let $u=\frac{q^{3 r}-1}{q^{r}-1}=q^{2 r}+q^{r}+1$ and let $D=\{i \in$ $\left.\mathbb{Z}_{u} \mid \alpha^{i} \in U\right\}$. Then by Singer's theorem [32], $D$ is a planar difference set in $\mathbb{Z}_{u}$.

Similarly, we may consider $\mathrm{GF}\left[q^{3}\right]$ as a 3 dimensional vector space over $\mathrm{GF}[q]$. In this space the vectors 1 and $\beta$ span a 2 dimensional subspace $W$. Let $w=\frac{q^{3}-1}{q-1}=q^{2}+q+1$ and let $T=\left\{i \in \mathbb{Z}_{w} \mid \beta^{i} \in W\right\}$. Again $T$ is a planar difference set in $\mathbb{Z}_{w}$. As $\frac{q^{r}-1}{q-1}$ and $w$ are coprime, multiplication by $\frac{q^{r}-1}{q-1}$ is an automorphism of $\mathbb{Z}_{w}$ and so $T^{\prime}=\left\{\frac{q^{r}-1}{q-1} i \mid i \in T\right\}$ is a planar difference set. Then the set $T^{\prime \prime}=\left\{\frac{q^{r}-1}{q-1} v i \mid i \in T\right\}$ is a difference set in subgroup $\langle v\rangle$ of $\mathbb{Z}_{u}$. This set satisfies $T^{\prime \prime}=\left\{i \in \mathbb{Z}_{u} \mid \alpha^{i} \in W\right\}$ and $T^{\prime \prime} \subset D$, as $\beta=\alpha^{\frac{q^{r}-1}{q-1} v}$.

If for some $x, y \in D$ the difference $x-y$ is a non-zero multiple of $v$ then $x, y \in T^{\prime \prime}$. Let $D^{\prime}=D \backslash T^{\prime \prime}$. Let $S \subset \mathbb{Z}_{v}$ be the numbers congruent to numbers in $D^{\prime}$ modulo $v$. As multiples of $v$ are not differences in $D^{\prime},|S|=q^{r}-q$.

Let $g \in \mathbb{Z}_{v}, g \neq 0$. Then $g$ is congruent modulo $v$ to $q^{2}+q+1$ elements in $\mathbb{Z}_{u}$, each of which can uniquely be written as difference $x-y$ where $x, y \in D$. If $g \in S$ then exactly $q+1$ of these differences satisfy $y \in T^{\prime \prime}$. If $g \notin S$ then none of the differences have $y \in T^{\prime \prime}$. Similarly, if $g \in-S$ then exactly $q+1$ of the differences have $x \in T^{\prime \prime}$. 
Thus the number of pairs $x, y \in S$ such that a nonzero element $g \in \mathbb{Z}_{v}$ can be written as $g=x-y$ is $\mu=q^{2}+q+1$ if $g \notin S \cup-S, \lambda=q^{2}$ if $g$ is in exactly one of the sets $S,-S$, and $2 \lambda-\mu=q^{2}-q-1$ if $g \in S \cap-S$.

For $r=2$ the graph constructed in this theorem is a complete graph with $v=\frac{q^{4}+q^{2}+1}{q^{2}+q+1}=$ $q^{2}-q+1$. If $r \geqslant 4$ is even then the projective plane of order $q^{r}$ has an ordinary partition in subplanes of order $q^{2}$ and the planes of order $q^{2}$ have an ordinary partition in subplanes of order $q$. Then the vertices of the normally regular digraph are partitioned in sets of size $q^{2}-q+1$ spanning complete subgraphs. Thus $S \cap-S$ contains all nonzero elements of the subgroup of order $q^{2}-q+1$.

We conjecture that these are the only elements in $S \cap-S$.

Conjecture 2. Let $S$ be as in Theorem 36 .

- If $r$ is odd then $S \cap-S=\varnothing$.

- If $r$ is even then $S \cap-S$ consists of the nonzero elements of the subgroup of order $q^{2}-q+1$.

The proof of Theorem 36 is an algorithm for computing the set $S$. The above conjecture is based on computations of $S$ for the following values of $(q, r):(2,4),(2,5),(2,7),(2,8)$, $(2,10),(3,4),(3,5),(4,4),(4,5),(5,4)$.

Example 6. 1. For $q=2$ and $r=4$, we get

$$
S=\{7,13,14,17,19,23,26,28,29,31,34,35,37,38\} \in \mathbb{Z}_{39} .
$$

This gives an $N R D(39,14,4,7)$. In this particular case, the normally regular digraph is isomorphic to the graph constructed in Corollary 40, with $(s, t)=(0,3)$.

2. For $q=2$ and $r=5$, we get

$$
\begin{aligned}
S= & \{11,17,21,22,25,29,31,34,42,43,44,45,49,50,58,62, \\
& 68,81,84,86,88,90,91,97,98,100,116,121,124,136\} \in \mathbb{Z}_{151} .
\end{aligned}
$$

This gives an asymmetric $N R D(151,30,4,7)$.

\subsection{Product constructions}

In this section we give two constructions of normally regular digraphs that are not asymmetric. They are products involving doubly regular tournament and conference graphs. In some cases they are Cayley graphs.

Theorem 37. Let $T$ be a doubly regular tournament with $4 t+3$ vertices and let $K_{2 t+1}$ be the complete graph of order $2 t+1$.

Then the cartesian product with vertex set $V(T) \times V\left(K_{2 t+1}\right)$ and edge set

$$
\{(x, u) \rightarrow(y, u) \mid x \rightarrow y \text { in } T\} \cup\left\{(x, u) \leftrightarrow(x, v) \mid u \leftrightarrow v \text { in } K_{2 t+1}\right\}
$$

is an $N R D((4 t+3)(2 t+1), 4 t+1, t, 1)$. 
Proof. Let $(x, u)$ and $(y, v)$ be vertices in the cartesian product. If $x=y$ and $u \neq v$ then $(x, u)$ and $(y, v)$ are joined by an undirected edge and their common out-neighbours are the remaining $2 t-1$ vertices of the form $(x, w)$. If $x \neq y$ and $u=v$ then $(x, u)$ and $(y, v)$ are joined by a directed edge and their common out-neighbours are the vertices $(z, u)$ where $z$ is a common out-neighbour of $x$ and $y$ in $T$. There are $t$ such vertices. If $x \neq y$ and $u \neq v$ then $(x, u)$ and $(y, v)$ are non-adjacent. We may assume $x \rightarrow y$ in $T$. Then $(y, u)$ is the unique common out-neighbour of $(x, u)$ and $(y, v)$.

If $T$ is a Paley tournament then the above construction is a Cayley graph.

Corollary 38. Let $\mathbb{F}$ be the field of $4 t+3$ elements and let $Q$ be the set of non-zero squares in $\mathbb{F}$. Let $S=\{(d, 0) \mid d \in Q\} \cup\{(0, z) \mid z \neq 0\}$ be a subset of the direct product $\mathbb{F} \times \mathbb{Z}_{2 t+1}$ of the additive group of $\mathbb{F}$ and the cyclic group of order $2 t+1$.

Then $\operatorname{Cay}\left(\mathbb{F} \times \mathbb{Z}_{2 t+1}, S\right)$ is an $N R D((4 t+3)(2 t+1), 4 t+1, t, 1)$.

A strongly regular with $(v, k, \lambda, \mu)=(4 \mu+1,2 \mu, \mu-1, \mu)$ is called a conference graph. The most important construction of conference graphs are the Paley graphs which are constructed as follows. Let $\mathbb{F}$ be the field of $q$ elements, $q \equiv 1 \bmod 4$ and let $Q$ be the non-zero squares in $\mathbb{F}$. Then the Cayley graph of the additive group $\operatorname{Cay}(\mathbb{F}, Q)$ is a conference graph, see [7].

Theorem 39. Let $H$ be a conference graph with $4 t+1$ vertices and let $T$ be a doubly regular tournament with $4 s+3$ vertices. Let $G$ be the graph with vertex set $V(H) \times V(T)$ and with edge set

$$
\begin{aligned}
\{(u, x) \rightarrow & (v, y) \mid \text { either } u \leftrightarrow v \text { and } x \rightarrow y, \text { or } u \nLeftarrow \rightarrow v \text { and } x \leftarrow y\} \\
& \cup\{(u, x) \leftrightarrow(u, y) \mid u \in V(H), x, y \in V(T)\} .
\end{aligned}
$$

Then $G$ is a normally regular digraph with parameters

$$
((4 t+1)(4 s+3),(4 t+2)(2 s+1), 4 t s+3 s+t+1,(2 t+1)(2 s+1)) .
$$

Proof. Suppose that $(u, x) \rightarrow(v, y)$ but $(u, x) \nLeftarrow(v, y)$ in $G$. Then either $u \leftrightarrow v$ and $x \rightarrow y$, or $u$ and $v$ are non-adjacent and $x \leftarrow y$. Suppose that $u \leftrightarrow v$ and $x \rightarrow y$. Let $(w, z)$ be a common out-neighbour of $(u, x)$ and $(v, y)$. Then $w$ and $z$ satisfy one of the following six cases.

$$
\begin{gathered}
w=u, y \rightarrow z, \\
w=v, x \rightarrow z, z \neq y, \\
u \leftrightarrow w \leftrightarrow v, x \rightarrow z, y \rightarrow z, \\
u \leftrightarrow w \nLeftarrow \succ v, x \leftarrow z, y \leftarrow z, \\
u \leftrightarrow w \nLeftarrow \succ v, x \rightarrow z, y \leftarrow z, \\
u \leftrightarrow \leftrightarrow w \leftrightarrow v, x \leftarrow z, y \rightarrow z .
\end{gathered}
$$


The number of vertices $(w, z)$ in each case are $2 s+1,2 s, t s,(t-1) s$, ts and $t(s+1)$, respectively. The case where $u$ and $v$ are non-adjacent and $x \leftarrow y$ is similar. Thus $\lambda=4 t s+3 s+t+1$.

The parameters $k$ and $\mu$ are easy to compute. Now suppose that $(u, x) \leftrightarrow(v, y)$. Then $u=v$. A common out-neighbour $(w, z)$ of $(u, x)$ and $(u, y)$ is of one of the following three cases.

$$
\begin{gathered}
w=u, z \neq x, y, \\
w \leftrightarrow u, x \rightarrow z, y \rightarrow z, \\
w \nLeftarrow \rightarrow, x \leftarrow z, y \leftarrow z .
\end{gathered}
$$

The number of vertices of each type is $4 s+1,2 t s$ and $2 t s$, respectively. This adds up to $4 t s+4 s+1=2 \lambda-\mu$.

If the conference graph and the doubly regular tournament in this theorem are both of Paley type then $G$ is a Cayley graph.

Corollary 40. Let $\mathbb{F}$ and $\mathbb{E}$ be finite fields of order $4 t+1$ and $4 s+3$, respectively. Let $Q_{\mathbb{F}}$ and $Q_{\mathbb{E}}$ be the sets of non-zero squares in $\mathbb{F}$ and $\mathbb{E}$, respectively. Let $R_{\mathbb{F}}=\mathbb{F} \backslash\left(Q_{\mathbb{F}} \cup\{0\}\right)$ and $R_{\mathbb{E}}=\mathbb{E} \backslash\left(Q_{\mathbb{E}} \cup\{0\}\right)$. Let $S=\left(Q_{\mathbb{F}} \times Q_{\mathbb{E}}\right) \cup\left(R_{\mathbb{F}} \times R_{\mathbb{E}}\right) \cup(\{0\} \times(\mathbb{E} \backslash\{0\}))$. Then $\operatorname{Cay}(\mathbb{F} \times \mathbb{E}, S)$ is an $N R D((4 t+1)(4 s+3),(4 t+2)(2 s+1), 4 t s+3 s+t+1,(2 t+1)(2 s+1))$.

Note that if $(4 t+1)-(4 s+3)= \pm 2$ then we get the difference set with Hadamard parameters constructed by Stanton and Sprott [33] by adding $(0,0)$ to $S$ if $(4 t+1)-(4 s+$ $3)=2$ or by taking the complement of $S$ if $(4 t+1)-(4 s+3)=-2$.

\section{Acknowledgement}

The author would like to thank the anonymous referee for many valuable comments, which were used in improving the final version of this paper.

\section{References}

[1] E. Bannai and T. Ito. Algebraic Combinatorics. I. Benjamin/Cumming, Menlo Park, 1984.

[2] T. Beth, D. Jungnickel and H. Lenz. Design Theory. (Bibliographisches Institut, 1985 and) Cambridge University Press, 1993.

[3] R. M. Damerell. Distance-transitive and distance-regular digraphs. J. Combin. Th. Ser. B 31:46-53, 1981

[4] C. Delorme, L. K. Jørgensen, M. Miller and G. Pineda-Villavicencio. On bipartite graphs of diameter 3 and defect 2. J. Graph Th. 61:271-288, 2009

[5] A. M. Duval. A Directed Version of Strongly Regular Graphs. J. Combin. Th. Ser. A 47:71-100, 1972 
[6] M. Fossorier, J. Ježek,J. B. Nation and A. Pogel. Ordinary graphs and subplane partitions. Discrete Math. 282:137-148, 2004

[7] C. Godsil and G. Royle. Algebraic Graph Theory. Graduate Texts in Mathematics 207, Springer, 2001.

[8] R. W. Goldbach and H. L. Claasen. Feasibility conditions for non-symmetric 3-class association schemes. Discrete Math. 159:111-118, 1996.

[9] R. W. Goldbach and H. L. Claasen. The structure of imprimitive non-symmetric 3-class association schemes. Europ. J. Combin. 17:23-37, 1996.

[10] M. Hall, Jr. Combinatorial Theory, second edition. Wiley-Interscience, 1986.

[11] Y. Ionin and H. Kharaghani. Doubly regular digraphs and symmetric designs. J. Combin. Theory Ser. A 101:35-48, 2003.

[12] N. Ito. Doubly regular asymmetric digraphs. Discrete Math. 72:181-185, 1988.

[13] N. Ito. Automorphism groups of DRADs. Group theory (Singapore, 1987), de Gruyter, Berlin, 151-170, 1989.

[14] N. Ito. Doubly regular asymmetric digraphs with rank 5 automorphism groups. In Groups-Korea 1988), volume 1398 of Lecture Notes in Math., pages 94-99, Springer, 1989.

[15] N. Ito. On spectra of doubly regular asymmetric digraphs of RH-type. Graphs Combin. 5:229-234, 1989.

[16] N. Ito. On Hadamard Tournaments. J. Algebra 131:432-443, 1990.

[17] L. K. Jørgensen. Isomorphic switching in tournaments. Congressus Numerantium 104:217-222, 1994.

[18] L. K. Jørgensen. Non-existence of directed strongly regular graphs. Discrete Math. 264:111-126, 2003.

[19] L. K. Jørgensen. Algorithmic approach to non-symmetric 3-class association schemes. In: M. Klin et al. (eds.), Algorithmic algebraic combinatorics and Gröbner bases, pages 251-268, Springer, Berlin, 2009. doi: 10.1007/978-3-642-01960-9_8

[20] L. K. Jørgensen. Schur rings and non-symmetric association schemes on 64 vertices. Discr. Math. 310:3259-3266, 2010.

[21] L. K. Jørgensen. Normally regular digraphs: Abelian Cayley graphs. In preparation.

[22] L. K. Jørgensen. Normally regular digraphs: Enumeration of small graphs. In preparation.

[23] L. K. Jørgensen, G. A. Jones, M. H. Klin and S. Y. Song. Normally regular digraphs, association schemes and related combinatorial structures. Séminaire Lotharingien de Combinatoire, B71c: 39pp, 2014.

[24] A. Kotzig. Sur les tournois avec des 3-cycles régulière-ment placés. Mat. Casopis 19:126-134, 1969.

[25] R. A. Liebler and R. A. Mena. Certain Distance regular digraphs and related rings of characteristic 4. J. Combin. Theory Ser B 47:111-123, 1988. 
[26] Jianmin Ma and Kaishun Wang. For-class skew-symmetric association schemes. J. Combin. Th. Ser A 118:1381-1391, 2011.

[27] S. L. Ma.A survey of partial difference sets. Designs, Codes and Cryptography 4:221261, 1994.

[28] U. Ott. Cyclotomy and generalized difference sets. Invited talk at Combinatorics 2008 (Costermano (VR), Italy), 77-121.

[29] K. B. Reid and E. Brown. Doubly Regular Tournaments are Equivalent to Skew Hadamard Matrices. J. Combin. Theory Ser. A 12:332-338, 1988.

[30] M. J. de Resmini and D. Jungnickel. Two families of failed symmetric designs. Discrete Math. 261:235-241, 2003.

[31] P. Rowlinson. On 4-cycles and 5-cycles in regular tournaments. Bull. London Math. Soc. 18:135-139, 1986.

[32] J. Singer. A theorem in finite projective geometry and some applications to number theory. Trans. Amer. Math. Soc. 43:377-385, 1938.

[33] R. G. Stanton and D. A. Sprott. A family of difference sets. Canad. J. Math. 10:73-77, 1958.

[34] K. Wang and Y. Feng. Deza digraphs. Euro. J. Combin. 27 995-1004, 2006. 